\title{
Atuação do psicólogo na inclusão escolar de estudantes com autismo e deficiência intelectual
}

\author{
Priscila Benitez ${ }^{1}$, http://orcid.org/0000-0003-3501-7606
}

Camila Domeniconi², http://orcid.org/0000-0003-0486-3543

\begin{abstract}
Resumo
O objetivo do estudo foi operacionalizar a atuação do psicólogo-pesquisador no processo de inclusão escolar de estudantes com autismo (TEA) e deficiência intelectual (DI), a partir de intervenções aplicadas pelos professores e pais; e avaliar a aprendizagem de leitura e escrita do grupo experimental (exposto às intervenções) e controle. Participaram 14 estudantes, sete pais e nove professores. O procedimento envolveu o planejamento das intervenções, a capacitação com os agentes, aplicação das intervenções e supervisão pelo psicólogo, pós-teste e follow-up.Os desempenhos do grupo controle não apresentaram muitas variações entre pré e pós-teste. O grupo experimental passou de desempenho nulo para desempenhos maiores no pós-teste (leitura: 33\%, 75\% e 100\% de acertos para os demais; escrita: $50 \%, 75 \%, 75 \%$ e $100 \%$ de acertos para os demais). A atuação do psicólogo contribuiu com a inclusão dos estudantes com DI e TEA, a despeito das práticas segregadoras realizadas com esse público, em décadas anteriores.
\end{abstract}

Palavras-chave: Autismo; deficiência intelectual; inclusão escolar.

\section{Psychologist's activity in the school inclusion of students with autism and intellectual deficiency}

\begin{abstract}
The objective of the study was to operationalize the performance of the psychologist-researcher in the process of school inclusion of students with autism (ASD) and intellectual disability (DI), based on interventions applied by teachers and parents; and evaluate the reading and writing learning of the experimental group (exposed to interventions) and control. It was attended by 14 students, seven parents and nine teachers. The procedure involved the planning of the interventions, the training with the agents, the application of the interventions and supervision by the psychologist, post-test and follow-up. The performances of the control group did not present many variations between pre- and post-test. The experimental group went from zero to higher performance in the post-test (reading: $33 \%, 75 \%$ and $100 \%$ correct for others, written: $50 \%, 75 \%, 75 \%$ and $100 \%$ correct for the others). The performance of the psychologist contributed to the inclusion of students with ID and TEA, in spite of the segregating practices performed with this public, in previous decades.
\end{abstract}

Keywords: Autism; intellectual disability; school inclusion

\section{Actuación del psicólogo en la inclusión escolar de estudiantes con autismo y deficiencia intelectual}

\begin{abstract}
Resumen segregadoras realizadas con ese público, en décadas anteriores.

Palabras clave: Autismo; deficiencia intelectual; inclusión escolar.

1 Universidade Federal do ABC, Centro de Matemática, Computação e Cognição - Santo André, SP, Brasil.

2 Universidade Federal de São Carlos, Departamento de Psicologia - São Carlos, SP, Brasil.
\end{abstract}

El objetivo del estudio fue la operatividad la actuación del psicólogo-investigador en el proceso de inclusión escolar de estudiantes con autismo (TEA) y deficiencia intelectual (DI), a partir de intervenciones aplicadas por los profesores y padres; y evaluar el aprendizaje de lectura y escritura del grupo experimental (expuesto a las intervenciones) y control. Participaron 14 estudiantes, siete padres y nueve profesores. El procedimiento involucró la planificación de las intervenciones, la capacitación con los agentes, aplicación de las intervenciones y supervisión por el psicólogo, post-test y follow-up. Los desempeños del grupo control no presentaron muchas variaciones entre pre y post-test. El grupo experimental pasó de desempeño nulo para desempeños mayores en el post-test (lectura: el 33\%, el $75 \%$ y el $100 \%$ de aciertos para los demás; escritura: el $50 \%$, el $75 \%$, el $75 \%$ y el $100 \%$ de aciertos para los demás). La actuación del psicólogo contribuyó con la inclusión de los estudiantes con DI y TEA, a pesar de las prácticas 


\section{Introdução}

Com a deliberação dos documentos internacionais Educação para Todos (Unicef, 1990) e Declaração de Salamanca (Unesco, 1994), a educação inclusiva ganhou força no contexto educacional mundial e desde então, são discutidas formas de trabalho escolar que favoreçam a todos os estudantes e não apenas, uma única parcela. Diferentemente da integração escolar, a inclusão prevê modificações no ambiente que favoreçam a inclusão de todos os estudantes, a partir de um sistema de apoio, com suporte massivo de diferentes serviços, como, o acesso ao atendimento educacional especializado (Decreto $n^{\circ} 7.611,2011$ ), o trabalho conjunto entre os diferentes agentes educacionais (Benitez \& Domeniconi, 2015), o trabalho colaborativo do professor de educação especial em sala de aula com o estudante, em colaboração com o professor de sala (Vilaronga \& Mendes, 2014) e dentre outros suportes necessários para atingir o ensino e aprendizagem de todos os estudantes.

Nesse contexto, a inclusão escolar beneficia tanto o desempenho social do estudante, como o desempenho do repertório básico de leitura e escrita. Tais habilidades são fundamentais para a aprendizagem dos conteúdos acadêmicos durante a educação formal (Benitez \& Domeniconi, 2016; Reis, Souza, \& Rose, 2009) e importantes para a inserção de todos os estudantes na maioria das atividades realizadas em sociedade, o que demonstra um papel fundamental na independência e adaptação aos grupos sociais presentes em suas respectivas comunidades. Dada à importância destes repertórios e o que é previsto em lei (Brasil, 2008) todas as crianças, com e sem qualquer deficiência e/ ou transtorno, deveriam aprender a ler e escrever nos anos iniciais do ensino fundamental.

Pesquisas desenvolvidas no âmbito da Psicologia relacionadas à Educação Especial, visando o ensino de leitura e de escrita têm mostrado resultados positivos no ensino de leitura e escrita para estudantes com Transtorno do Espectro do Autismo (TEA) ${ }^{3}$, Deficiência Intelectual (DI) e demais populações (Benitez \& Domeniconi, 2016; Rose, Souza, \& Hanna, 1996; Melchiori, Souza, \& Rose, 2000; Sidman, 1971; Sidman, \& Cresson, 1973). Apesar das dificuldades encontradas pelo estudante com TEA, especialmente no que concerne a linguagem e comportamento social, quando as intervenções são bem planejadas as contingências de ensino e de reforçamento mostram resultados promissores no ensino de diferentes comportamentos específicos (Lovaas, 1987).

\footnotetext{
3 Dentre as principais características definidoras topograficamente do TEA, podem-se destacar: comprometimento qualitativo nas interações sociais, na comunicação e na linguagem. Grande parte de crianças assim diagnosticadas apresenta diminuto vocabulário, geralmente desprovido de função, sendo comum a ocorrência de ecolalia e mutismo (APA, 2013).

4 A deficiência intelectual (DI), conforme a American Associationon Intellectual and Developmental Disabilities (AAIDD) é compreendida como redução significativa no funcionamento intelectual e no comportamento adaptativo expresso em habilidades sociais, conceitos e práticas, oriundas antes dos 18 anos (Wehmeyer, 2003).
}

Os resultados do estudo de Benitez e Domeniconi (2016) evidenciaram que dos seis estudantes com DI, cinco aprenderam habilidades de leitura, com aumento considerável no pós-teste para leitura das palavras ensinadas diretamente, em média, de 5,32\% (pré-teste) a $89,32 \%$ (pós-teste) de acertos. O comportamento dos familiares enquanto aplicadores foi avaliado em termos de fornecimento de dicas ao estudante com DI e frequência de aplicação das sessões. O número de dicas reduziu conforme o estudante atingia os critérios de ensino programados para cada passo, o que indica que o estudante respondeu, cada vez mais, sob controle das instruções e tarefas fornecidas pelo programa informatizado, dispensando o uso de dicas extras, o que foi considerado adequado, assim como a frequência de aplicação das sessões (três vezes por semana). O estudo permitiu concluir que a residência, como local de aplicação e familiares como aplicadores podem configurar uma situação promissora de aprendizagem para essa população.

Em relação ao trabalho com os pais e professores conjuntamente, Benitez e Domeniconi (2014) operacionalizaram as etapas de uma capacitação destinada aos professores da sala de aula regular, professores da educação especial e pais para aplicarem atividades de ensino de leitura e escrita com estudantes com TEA e DI. A capacitação foi composta por três momentos. No primeiro, foi realizada uma discussão sobre as condutas dos agentes com os estudantes, durante a realização das sessões (em termos de níveis de ajuda que seriam consideradas adequadas e inadequadas), a partir de uma explanação teórica sobre as habilidades sociais educativas (Del Prette \& Del Prette, 2008). No segundo momento, foi abordada uma exposição teórica sobre as questões técnicas de aplicação de cada atividade de ensino, propostas para cada agente educacional, com a apresentação de um checklist com os comportamentos presentes para serem seguidos durante a aplicação. O terceiro momento foi baseado em um trabalho com cada agente, com a finalidade de instruir in lócus cada um deles, a partir de feedbacks dados para as condutas apresentadas durante a aplicação das atividades de ensino.

Dessa maneira, as capacitações citadas (Benitez \& Domeniconi, 2014, 2016) tiveram uma primeira parte composta por uma discussão teórica (o que não demonstra qualquer aspecto inovador, se comparado ao que é feito tradicionalmente junto aos professores) e uma segunda parte, composta pela supervisão individual do pesquisador com cada agente, na situação aplicada. Essa segunda parte demonstra um diferencial, no que concerne a sistematização da aplicação dos procedimentos de ensino, pois nesse momento o pesquisador tinha a oportunidade de observar a aplicação, fornecer modelos adequados de atuação e esclarecer dúvidas. Esse acompanhamento realizado pelo pesquisador, em detalhes, da evolução de cada díade (agente educacional-estudante) criou oportunidade para fornecer feedback constante, em tempo real e ratificar para o agente a importância da intervenção. A sistematização e o acompanhamento do trabalho parecem ser um foco promissor para a elaboração de futuras capacitações com os agentes. 
A presente proposta parte da premissa de que a inclusão escolar é um processo social complexo que envolve os diferentes agentes educacionais, inclusive o profissional da área da Psicologia, o qual pode contribuir com o trabalho conjunto entre os diferentes agentes educacionais, como o professor de sala de aula, da Educação Especial e os pais, como uma estratégia para o ensino dos comportamentos de ler e escrever dos estudantes com DI e TEA, matriculados na escola regular. Evidentemente, ao considerar as especificidades de cada agente educacional é necessário estabelecer diferentes práticas pedagógicas para o ensino de tais comportamentos, visando o contexto natural em que cada um deles está inserido. Os estudos de Benitez e Domeniconi (2014, 2016) mostraram a importância do trabalho conjunto entre o pesquisador e tais agentes educacionais, no entanto, tais estudos não compararam os efeitos da exposição do trabalho sistematizado aplicado pelos agentes, com supervisão do psicólogo-pesquisador, em relação à composição de um grupo experimental e controle.

Assim sendo, o presente estudo apresentou fundamentalmente dois objetivos, a destacar, a) operacionalizar a atuação do psicólogo-pesquisador no processo de inclusão escolar, a partir de intervenções aplicadas pelos professores (da sala de aula e da Educação Especial) e pais, como promotores do ensino de leitura e escrita com estudantes com TEA e DI; e b) avaliar a aprendizagem de leitura e escrita de dois grupos de estudantes com DI e TEA, sendo que o
Grupo Experimental foi exposto às intervenções oriundas do estudo e o Grupo Controle não foi exposto à intervenção.

\section{Método}

\section{Considerações éticas}

O projeto foi aprovado pelo Comitê de Ética em Pesquisa com Seres Humanos da UFSCar (CAAE 4918.0.000.135-10 - Parecer 081/2011).

\section{Participantes}

Sete crianças, seis professores de sala de aula e três professores de educação especial compuseram a amostra do grupo experimental e foram expostos às intervenções de ensino (A1 - A7). Outras sete crianças (A8 - A14) compuseram a amostra do grupo controle. A Tabela 1 contém características de ambos os grupos (porção superior da tabela os alunos do grupo experimental e na porção inferior os do grupo controle). O diagnóstico atribuído a todos os estudantes foi conduzido por uma equipe multidisciplinar de uma instituição especializada.

Tabela 1. Caracterização dos participantes.

\begin{tabular}{|c|c|c|c|c|c|c|c|c|c|c|c|c|c|}
\hline \multirow[t]{2}{*}{ Estudante } & \multirow[t]{2}{*}{ Idad } & \multirow[t]{2}{*}{ Diagn } & \multicolumn{4}{|c|}{ WISC-III } & \multirow[t]{2}{*}{ PPVT } & \multirow[t]{2}{*}{ Mãe } & \multirow[t]{2}{*}{ Idad } & \multirow[t]{2}{*}{ PSA } & \multirow[t]{2}{*}{ Idad } & \multirow[t]{2}{*}{ PEE } & \multirow{2}{*}{$\begin{array}{l}\text { Ida- } \\
\text { de }\end{array}$} \\
\hline & & & Verb & Exec & Total & Classificação & & & & & & & \\
\hline $\mathrm{A} 1-\hat{0}$ & $9^{a}$ & TEA & 50 & 69 & 56 & Intelectualmente deficiente & $2 a 6 m$ & M1 & 30 & PSA1 & 47 & PEE1 & 29 \\
\hline $\mathrm{A} 2-q$ & $10 a$ & DI & 55 & 53 & $<50$ & Intelectualmente deficiente & $3 a 4 m$ & M2 & 35 & PSA2 & 43 & PEE1 & 29 \\
\hline$A 3-\hat{O}$ & $11^{a}$ & TEA & 62 & 57 & 56 & Intelectualmente deficiente & $5 a 5 m$ & M3 & 56 & PSA3 & 41 & PEE1 & 29 \\
\hline $\mathrm{A} 4-q$ & $9^{a}$ & DI & 71 & 73 & 69 & Intelectualmente deficiente & $3 a 9 m$ & M4 & 35 & PSA4 & 37 & PEE2 & 24 \\
\hline $\mathrm{A} 5-q$ & $10^{\mathrm{a}}$ & DI & 47 & 53 & $<50$ & Intelectualmente deficiente & $3 a 7 m$ & M5 & 35 & PSA4 & 37 & PEE2 & 24 \\
\hline $\mathrm{A} 6-\hat{\sigma}$ & $10^{\mathrm{a}}$ & TEA & 51 & 49 & $<50$ & Intelectualmente deficiente & $3 a 5 m$ & M7 & 30 & PSA6 & 29 & PEE3 & 32 \\
\hline $\mathrm{A} 7-q$ & $9^{a}$ & DI & 56 & 66 & 57 & Intelectualmente deficiente & $3 a 5 m$ & M6 & 37 & PSA5 & 59 & PEE1 & 29 \\
\hline $\mathrm{A} 8-\hat{0}$ & $9^{a}$ & TEA & 52 & 79 & 62 & Intelectualmente deficiente & $3 a 8 m$ & - & - & - & - & - & - \\
\hline $\mathrm{A} 9-\hat{0}$ & $9^{a}$ & DI & 62 & 83 & 69 & Intelectualmente deficiente & $4 a 0 m$ & - & - & - & - & - & - \\
\hline $\mathrm{A} 10-q$ & $10^{\mathrm{a}}$ & TEA & 53 & 45 & 50 & Intelectualmente deficiente & $2 a 5 m$ & - & - & - & - & - & - \\
\hline $\mathrm{A} 11-q$ & $10^{\mathrm{a}}$ & DI & 59 & 57 & 54 & Intelectualmente deficiente & $5 a 1 m$ & - & - & - & - & - & - \\
\hline $\mathrm{A} 12-q$ & $10^{a}$ & DI & 75 & 76 & 75 & Limítrofe & $5 a 1 m$ & - & - & - & - & - & - \\
\hline $\mathrm{A} 13-\hat{\sigma}$ & $9^{a}$ & DI & 60 & 71 & 62 & Intelectualmente deficiente & $4 a 1 m$ & - & - & - & - & - & - \\
\hline $\mathrm{A} 14-\hat{o}$ & $11^{\mathrm{a}}$ & DI & 70 & 83 & 73 & Limítrofe & $7 a 8 m$ & - & - & - & - & - & - \\
\hline
\end{tabular}

Nota. PSA refere-se ao professor da sala de aula e PEE ao professor da Educação Especial. Na porção superior da tabela estão listados os aprendizes do grupo experimental e na porção inferior, com sombreamento, os participantes do grupo controle. 
Os critérios para a seleção dos estudantes, incluídos na rede regular de ensino foram: a) resultado igual ou inferior a 69 com a aplicação do WISC-III (Wechsler \& Figueiredo, 2002), b) resultado inferior a $20 \%$ de acertos na tarefa de nomeação de palavra impressa e de ditado por composição, conforme aplicação do pré-teste, c) resultados iguais ou superiores a $60 \%$ nos testes de emparelhamento por identidade com figuras e com palavras impressas (relações BB e CC, respectivamente) e emparelhamento palavra ditada-figura (relação $A B$ ) no pré-teste, d) estudantes matriculados nos anos iniciais do ensino fundamental (do $1^{\circ}$ ao $5^{\circ}$ ano), que apresentaram os seguintes comportamentos: contato visual quando chamado pelo nome, seguir instruções, sentar na cadeira e realizar uma atividade com lápis. Os critérios de seleção foram fundamentados no desempenho de cada um deles, avaliados por instrumentos comprovadamente relevantes para essa finalidade.

Com relação aos critérios para a seleção dos pais, foi sugerido que tivessem, no mínimo, a primeira etapa do ensino fundamental concluída e disponibilidade de participação. Para os professores da educação especial e da sala de aula, o critério foi a disponibilidade de participação.

Em relação ao histórico de saúde dos sete estudantes, A1, A3 e A6 eram expostos ao tratamento medicamentoso. Os demais não eram expostos a tal tratamento e, de acordo, com os relatos de seus pais não apresentavam alterações neurológicas e/ou metabólicas, ou associações a síndromes.

Os estudantes do grupo controle (porção inferior da Tabela 1) foram expostos apenas ao pré e ao pós-teste, sem qualquer exposição à intervenção de ensino pelos agentes.

O tempo médio de duração do estudo foi calculado por dias corridos, ou seja, o primeiro dia se referiu à apli- cação da primeira avaliação e o último dia à aplicação da última avaliação. A coleta de dados com cada estudante do grupo experimental foi equivalente ao mínimo de três meses e ao máximo de quatro meses. Foram calculados os dias de duração do estudo para cada estudante e dividido por sete, a fim de obter um tempo médio entre eles. O resultado foi aproximado para 110 dias. Assim, o tempo de espera entre uma avaliação (medida de pré-teste) e a outra (medida de pós-teste) do grupo controle foi 110 dias. Todos os participantes do grupo controle foram, posteriormente a este estudo, inseridos em intervenções com agentes educacionais isolados (ou com pais, ou com professores de sala de aula ou com professores da educação especial), utilizando os mesmo materiais e procedimentos utilizados com o grupo experimental do presente estudo.

\section{Procedimento}

\section{Etapa 1: Planejamento do procedimento de ensino das três intervenções}

Esse item descreve a estrutura do programa de ensino, dividida em: a) pré e pós-teste, b) medidas antes e após o ensino do conjunto de sete palavras (Pré e Pós), c) medidas entre condição experimental (Teste). $O$ ensino é compreendido como a exposição do estudante por todas as sete condições experimentais. Cada palavra foi ensinada em uma condição experimental específica. Cada condição era composta por 30 atividades. A Figura 1 exemplifica a trajetória percorrida pelos estudantes que compuseram o Grupo Controle

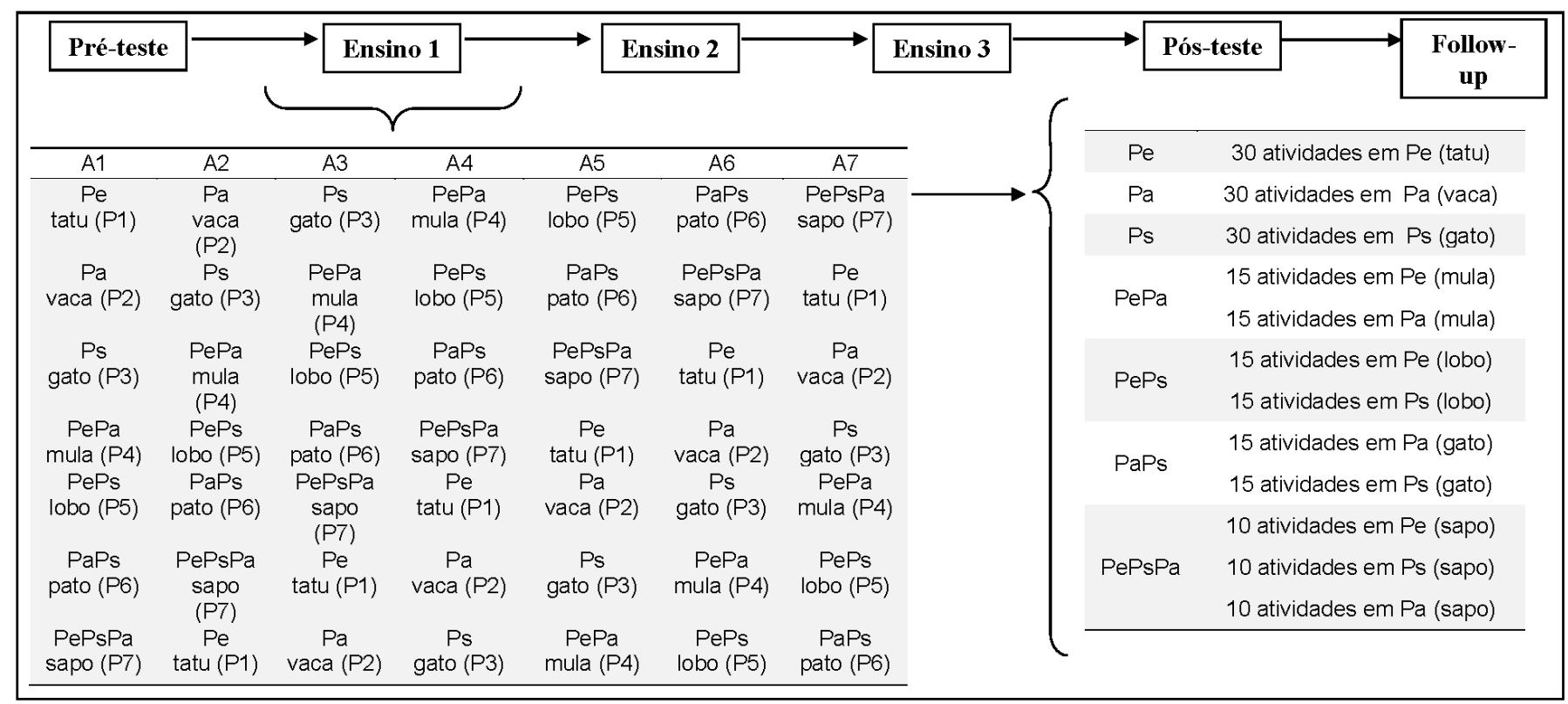

Figura 1. Trajetória dos estudantes do Grupo Experimental ao longo dos três ensinos. P1 significa Palavra 1, P2 palavra 2 e assim por diante, Pré1 e Pós1 significam medidas aplicadas antes e após cada ensino (conjuntos de sete palavras), Teste foi aplicado entre condição experimental, Pe intervenção aplicada pelo professor de educação especial, Pa aplicada pelos pais e Ps pelo professor de sala de aula. 


\section{Pré-teste, pós-teste e follow-up}

O pré, pós-teste e follow-up foram compostos por 15 atividades $^{5}$, divididas em seis de seleção (relações $B B, C C$, $A B, A C, B C, C B$ ) e nove de execução ( $C D$ - dividida em nomeação de palavra inteira (CDp), de sílabas (CDs), de consoantes (CDc) e de vogais (CDv), BD, AE, AF, CE, CF). Cada atividade foi apresentada 10 vezes, envolvendo palavras diferentes: quatro palavras que foram ensinadas posteriormente (três com duas sílabas e uma com três sílabas), três de generalização (que não foram ensinadas diretamente e eram formadas pelas sílabas das palavras treinadas) e três extras (que não foram ensinadas diretamente e eram compostas por sílabas de palavras que não foram treinadas).

As sílabas testadas na tarefa de nomeação de sílabas eram: bo, te, sa, pe, fi, ma, ta, ni, da, co, go, su, ti, le, ve, me, no, la, tu, ja. As atividades não continham consequências diferenciais para acerto ou erro. A estrutura adotada no pré, pós-teste e follow-up envolveu um conjunto de tarefas que avaliaram os pré-requisitos, leitura e escrita (Souza, Rose, \& Domeniconi, 2009), com um total de 10 palavras.

É importante salientar que foram escolhidas seis palavras do Ensino 1, seis do Ensino 2 e quatro do Ensino 3. Tanto o pré, quanto o pós-teste e o follow-up foram divididos em três sessões. Na primeira foram avaliadas as tarefas:

5 Legenda: BB - emparelhamento por identidade com figuras, CC - emparelhamento por identidade com palavras impressas, AB seleção de figura diante da palavra ditada, $A C$ - seleção da palavra impressa diante da palavra ditada, BC - seleção da palavra impressa diante da figura, CB - seleção da figura diante da palavra impressa, $\mathrm{BD}$ - nomeação da figura, CDp - leitura da palavra impressa, CDs nomeação de sílaba, CDc - nomeação de consoante, CDv - nomeação de vogal, AE - ditado por composição, AF - ditado manuscrito, CE cópia por composição, CF - cópia manuscrita.
$B B, A C, C D, B D, C D v, C D c$. Na segunda: $B C, C E, A E, A B . E$ na terceira: CDs, CC, AF, CF, CB.

\section{Estrutura geral de cada intervenção}

A Tabela 2 descreve as atividades aplicadas em cada intervenção.

Todas as intervenções tinham quatro etapas: a) Pré-teste com a tarefa de emparelhamento entre palavra ditada e palavra impressa (relação AC), b) Ensino, com o professor da educação especial, o ensino foi aplicado a partir do programa informatizado elaborado com base no currículo suplementar (de Souza \& cols., 2009) e com o professor da sala de aula, a partir de um texto lido oralmente para todos os estudantes e seguido de questões de compreensão sobre a história; com os pais, por meio da leitura individualizada de um livro, seguido das questões; c) Pós-teste composto pela mesma tarefa do pré-teste; d) Ensino adicional com tarefas de escrita (cópia e ditado) e de seleção de sílabas, a partir da sílaba ditada. É importante salientar que nessas tarefas não foram utilizados quaisquer critérios de aprendizagem, ou seja, o estudante prosseguia para a próxima tarefa independente do seu desempenho.

A condição Pe era composta por 24 atividades de emparelhamento palavra ditada-palavra impressa, três de cópia por composição e três de ditado por composição. Além disso, as condições $\mathrm{Pa}$ e Ps eram compostas por 30 frases e seguidas de questões, sendo que na condição $\mathrm{Pa}$ tinha uma figura correspondente para cada frase. Todas eram seguidas do ensino adicional. A condição PePa era dividida em Pe (composta por 15 atividades de palavra ditada-palavra impressa, três de cópia por composição e três de ditado por composição) e Pa (composta por 15 frases e cada uma delas tinha

Tabela 2. Descrição das atividades aplicadas em cada intervenção.

\begin{tabular}{|c|c|c|c|c|c|c|c|c|c|}
\hline & \multicolumn{3}{|c|}{$\begin{array}{c}\text { Intervenção Pe } \\
\text { Professor de educação especial }\end{array}$} & \multicolumn{3}{|c|}{$\begin{array}{l}\text { Intervenção Ps } \\
\text { Professor da sala de aula }\end{array}$} & \multicolumn{3}{|c|}{$\begin{array}{l}\text { Intervenção Pa } \\
\text { Pais }\end{array}$} \\
\hline & Tarefa $^{1}$ & Material & Aplicador & Tarefa & Material & Aplicador & Tarefa & Material & Aplicador \\
\hline Pré-teste & $A C$ & $\begin{array}{l}\text { Currículo su- } \\
\text { p I e m e n t a r } \\
\text { adaptado }\end{array}$ & PEE & $\mathrm{AC}$ & $\begin{array}{l}\text { Ficha im- } \\
\text { pressa }\end{array}$ & Pesquisador & $\mathrm{AC}$ & $\begin{array}{l}\text { Ficha im- } \\
\text { pressa }\end{array}$ & $\begin{array}{l}\text { Pesquisa- } \\
\text { dor }\end{array}$ \\
\hline Ensino & $\begin{array}{l}\mathrm{AC}, \mathrm{CE}, \\
\mathrm{AE}\end{array}$ & $\begin{array}{l}\text { Currículo su- } \\
\text { p le me n t a r } \\
\text { adaptado }\end{array}$ & PEE & $\begin{array}{l}\text { Leitura, } \\
A C, Q C\end{array}$ & $\begin{array}{l}\text { Texto im- } \\
\text { presso }\end{array}$ & PSA & $\begin{array}{l}\text { Leitura, } \\
A C, Q C\end{array}$ & $\begin{array}{l}\text { Livro im- } \\
\text { presso }\end{array}$ & Mãe \\
\hline Pós-teste & $A C$ & $\begin{array}{l}\text { Currículo su- } \\
\text { p le me n t a r } \\
\text { adaptado }\end{array}$ & PEE & $A C$ & $\begin{array}{l}\text { Ficha im- } \\
\text { pressa }\end{array}$ & Pesquisador & $A C$ & $\begin{array}{l}\text { Ficha im- } \\
\text { pressa }\end{array}$ & $\begin{array}{l}\text { Pesquisa- } \\
\text { dor }\end{array}$ \\
\hline $\begin{array}{l}\text { E n s i n o } \\
\text { adicional }\end{array}$ & $\begin{array}{l}\text { AEs, } A B, \\
C E S \\
B E \\
\text { AsCs }\end{array}$ & $\begin{array}{l}\text { Currículo su- } \\
\text { p l e m e n t a r } \\
\text { adaptado }\end{array}$ & PEE & $\begin{array}{l}\text { AEs, } A B, \\
C E \\
B \quad E \\
\text { AsCs }\end{array}$ & $\begin{array}{l}\text { Ficha im- } \\
\text { pressa }\end{array}$ & Pesquisador & $\begin{array}{l}\text { AEs, AB, } \\
C E \text { s , } \\
B E \text { s , } \\
\text { AsCs }\end{array}$ & $\begin{array}{l}\text { Ficha im- } \\
\text { pressa }\end{array}$ & $\begin{array}{l}\text { Pesquisa- } \\
\text { dor }\end{array}$ \\
\hline
\end{tabular}


uma figura correspondente, acompanhada de questões). A condição PePs, por sua vez, era formada por Pe (composta por nove atividades de palavra ditada-palavra impressa, três de cópia por composição e três de ditado por composição) e Ps (composta por 15 frases, seguida de questões). A condição PaPs era racionada em $\mathrm{Pa}$ (por 15 frases e uma figura correspondente para cada frase e questões) e Ps (por 15 frases e questões). Por fim, a condição PePsPa era dividida em $\mathrm{Pe}$ (composta por seis tarefas de palavra ditada-palavra impressa, duas de cópia por composição e duas de ditado por composição), $\mathrm{Pa}$ (por 10 frases e uma figura correspondente, seguida de questões) e Ps (10 frases com questões).

A Tabela 3 mostra o programa de ensino, distribuído pelas palavras que foram ensinadas diretamente pelos agentes, assim como aquelas que foram apenas avaliadas (generalização e extra).

\section{Etapa 2: Capacitação geral com os agentes educacionais}

Ocorreram três capacitações na escola: com os professores da sala de aula, da educação especial e com os pais. Primeiro, a estrutura de cada intervenção foi-lhes apresentada, em seguida, eles receberam o material de aplicação e, após breve exposição teórica, simulou-se uma aplicação de atividade, uns com os outros, alguns deles representando o papel dos estudantes.

Uma aplicação seria considerada como satisfatória, quando o agente seguisse os passos de cada aplicação e também apresentassem os seguintes comportamentos: a) fornecer a instrução da tarefa, b) elogiar/incentivar as respostas corretas, c) não apontar erros na resposta do estudante, d) não responder pelo estudante. Esses comportamentos foram considerados como importantes para a aplicação das sessões, no sentido de promover uma interação entre o agente e o estudante, que favorecesse o comportamento de estudar do estudante, com base nos argumentos de Benitez e Domeniconi (2012). Caso os agentes apresentassem os comportamentos (c) e (d), a pesquisadora fornecia o modelo de como poderiam interagir com o estudante, a partir do emprego dos comportamentos (a) e (b).

A função dos agentes variou conforme cada intervenção. O professor de Educação Especial tinha como função acompanhar a aplicação da sessão no programa de ensino informatizado (versão adaptada do currículo suplementar), ou seja, preparar a sessão no computador, convidar o estudante para realizá-la, acompanhar a aplicação e finalizá-la. Os pais foram instruídos a aplicarem a intervenção da seguinte forma: a) ler em voz alta cada frase do livro, b) solicitar que o estudante apontasse a palavra de ensino após a leitura de cada frase e, c) realizar as questões de compreensão. Durante as atividades na escola, o professor da sala de aula tinha como função ler em voz alta o texto sobre a palavra de ensino e realizar as questões de compreensão para toda a sala de aula. Na intervenção da sala de aula, o psicólogo-pesquisador sentava-se ao lado do estudante, com o texto impresso em cima da carteira e, conforme a professora lia em voz alta as palavras do texto, o psicólogo-pesquisador passava o dedo na palavra impressa correspondente, com o objetivo de garantir as respostas de observação do estudante. A capacitação dos agentes foi detalhadamente descrita em Benitez e Domeniconi (2014).

\section{Etapas 3 e 4: Aplicação das intervenções e Supervisão}

As intervenções foram aplicadas por cada agente e, simultaneamente a elas, ocorreu a Etapa 4 (Supervisão). Para aplicação das intervenções, eram realizadas supervisões com cada agente, nas residências e nas escolas, ao menos três vezes por semana. A supervisão consistia em instruir o agente a evitar fornecer dica excessiva ao estu-

Tabela 3. Distribuição das palavras de ensino $(P 1, P 2, P 3, P 4, P 5, P 6$ e $P 7)$ empregadas em cada intervenção.

\begin{tabular}{|c|c|c|c|c|c|c|c|c|c|}
\hline & P1 & P2 & P3 & P4 & P5 & P6 & P7 & Generaliz. & Extra \\
\hline & $\mathrm{Pe}$ & $\mathrm{Pa}$ & Os & $\mathrm{PePa}$ & PePs & PaPs & PePsPa & & \\
\hline Pré-teste & \multicolumn{7}{|c|}{$\begin{array}{l}\text { tatu, vaca, gato, lobo, pato, sapo, dedo, caju, figo, suco, bolo, vela, tapete, janela, } \\
\text { camelo, cavalo }\end{array}$} & $\begin{array}{l}\text { bola, mapa, sacola, } \\
\text { boca, galo, panela, } \\
\text { fila, mato, lago, } \\
\text { mala, fita, caneta }\end{array}$ & $\begin{array}{l}\text { lima, lupa, salada, } \\
\text { luva, bule, bigode, } \\
\text { fogo, fada, muleta, } \\
\text { sino, pipa, tomada }\end{array}$ \\
\hline Ensino 1 & tatu & vaca & gato & mula & lobo & pato & sapo & - & - \\
\hline Ensino 2 & dedo & caju & figo & suco & bolo & vela & faca & - & - \\
\hline Ensino 3 & tapete & janela & caneca & menina & camelo & cavalo & macaco & - & - \\
\hline $\begin{array}{l}\text { Pós-teste e } \\
\text { Follow-up }\end{array}$ & \multicolumn{7}{|c|}{$\begin{array}{l}\text { tatu, vaca, gato, lobo, pato, sapo, dedo, caju, figo, suco, bolo, vela, tapete, janela, } \\
\text { camelo, cavalo }\end{array}$} & $\begin{array}{l}\text { bola, mapa, sacola, } \\
\text { boca, galo, panela, } \\
\text { fila, mato, lago, } \\
\text { mala, fita, caneta }\end{array}$ & $\begin{array}{l}\text { lima, lupa, salada, } \\
\text { luva, bule, bigode, } \\
\text { fogo, fada, muleta, } \\
\text { sino, pipa, tomada }\end{array}$ \\
\hline
\end{tabular}




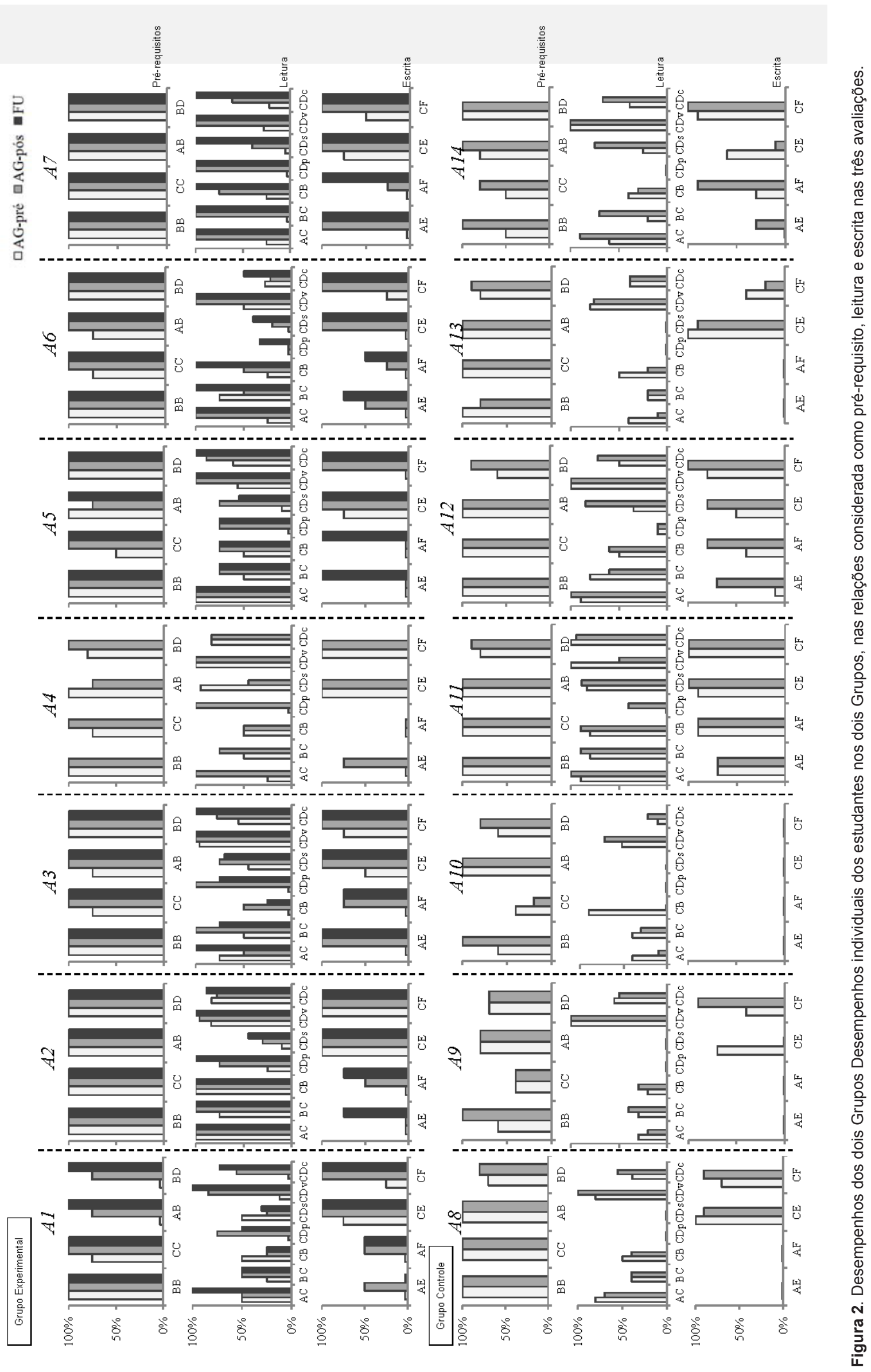


dante, evitar conversar sobre outros assuntos durante aplicação, minimizar barulhos e interferências externas e evitar consequenciar negativamente o desempenho do estudante em caso de erro ou desatenção.

\section{Etapa 5 e 6: Aplicação do pós-teste e follow-up}

Ao finalizar a aplicação dos três Ensinos, foi aplicado o pós-teste e o follow-up (similar ao pré-teste) para verificar se cada estudante apresentou progresso nas tarefas de leitura e escrita, em relação aos seus respectivos repertórios de entrada. Os testes foram aplicados também com o grupo controle.

\section{Análise de dados}

Os dados foram analisados a partir dos desempenhos individuais de cada estudante, distribuídos em cada grupo (Experimental e Controle), nas diferentes tarefas avaliadas durante o pré, pós-teste e medida de follow-up.

\section{Resultados}

A Figura 2 sumariza os desempenhos do Grupo Experimental e Controle em tarefas de pré-requisitos, leitura e escrita. Os dados de leitura (com análise simplificada pela relação CDp - nomeação de palavra impressa) e escrita (relação AE - ditado por composição) mostraram uma diferença entre o Grupo Experimental e o Grupo Controle, comparando as medidas de pré-teste com as porcentagens de acertos no pós-teste.

Na leitura, todos os estudantes do Grupo Experimental apresentaram desempenhos nulos na avaliação inicial e na avaliação final responderam corretamente com diferenças entre os desempenhos individuais. A6 (passou de desempenho nulo para $33 \%$ de acertos); A1 e A5 (passaram de desempenho nulo para $75 \%$ de acertos) e A2, A3, A4, A7 (de desempenho nulo para $100 \%$ de acertos). Os estudantes do Grupo Controle, por sua vez, não apresentaram muitas diferenças entre as porcentagens de acertos entre as medidas de pré e pós-teste. Para leitura, apenas A11 demonstrou variação entre as medidas, passando de desempenho nulo para $40 \%$ de acertos no pós-teste; enquanto os demais (A8, A9, A10, A13 e A14) mantiveram os mesmos desempenhos.

$\mathrm{Na}$ escrita, os estudantes do Grupo Experimental mantiveram o mesmo padrão do desempenho em leitura, uma vez que todos eles apresentaram desempenho nulo no pré-teste e no pós-teste apresentaram desempenhos iguais ou superiores a $50 \%$ de acertos. A1 obteve $50 \%$ de acertos no pós-teste; $A 4$ e $A 6$ atingiram $75 \%$ de acertos e os demais $100 \%$ de acertos (A2, A3, A5, A7). Os estudantes do Grupo Controle replicaram os dados de desempenho de leitura nas atividades de escrita, visto que a maioria deles (A8, A9, A10, A11 A13) manteve os mesmos padrões de acertos e erros nas duas avaliações, com exceção de A12 (que respondeu corretamente $10 \%$ no pré e $70 \%$ no pós-teste) e A14 (de desempenho nulo no pré, respondeu corretamente $30 \%$, no pós-teste).
Em relação à atuação do psicólogo-pesquisador em cada fase do procedimento, ao longo de todo o estudo, tal profissional acompanhou todas as etapas de aplicação do procedimento, com todos os sete estudantes.

\section{Discussão}

O estudo avaliou a aprendizagem de leitura e escrita de dois grupos de estudantes com DI e TEA, sendo que um grupo foi exposto às intervenções aplicadas pelos agentes educacionais e outro grupo não foi exposto às intervenções. O método utilizado e as discussões que podem ser conduzidas a partir dos resultados de aprendizagem dos estudantes possibilitam destacar alguns elementos importantes da atuação do psicólogo-pesquisador no processo de inclusão escolar, como apoio e suporte às intervenções aplicadas pelos professores (da sala de aula e da Educação Especial) e pais, enquanto promotores do ensino de leitura e escrita com estudantes com TEA e DI.

A despeito dos esforços empreendidos para que todos os estudantes, inclusive com DI e TEA, estejam inseridos na rede regular de ensino (Brasil, 2008), poucos são os estudos que trabalharam o ensino de repertórios acadêmicos, como a leitura e a escrita (Benitez \& Domeniconi, 2015; Miguel, Yang, Finn, \& Ahearn, 2009). Aparentemente, apesar das dificuldades na linguagem expressiva e receptiva, estudantes com TEA expostos aos procedimentos de ensino individualizado e personalizado, que atendam suas necessidades específicas aprendem a nomear palavras impressas, mesmo antes de desenvolverem a linguagem falada (Baldaçara, Nobrega, Leonard, \& Tengan, 2006) o que aponta a possibilidade de que eles aprendam a leitura de palavras impressas, uma vez programadas contingências adequadas de ensino, como têm acontecido com estudantes com diferentes repertórios.

No que concerne ao desempenho dos estudantes do presente estudo nas tarefas de leitura, o Grupo Experimental apresentou maior desempenho no pós-teste $(75 \%, 100 \%$, $100 \%, 100 \%, 75 \%, 33 \%$ e $100 \%$ ), ao comparar os respectivos desempenhos nulos de todos eles, no pré-teste. Os estudantes do Grupo Controle, com exceção de A11, mantiveram os mesmos desempenhos nas duas avaliações. E, em relação, aos dados de escrita, os estudantes do Grupo Experimental apresentaram desempenhos nulos no pré-teste, com desempenhos maiores no pós-teste $(50 \%, 100 \%$, $100 \%, 75 \%, 100 \%, 75 \%$ e $100 \%$ ). Enquanto os estudantes do Grupo Controle mantiveram os mesmos desempenhos nas duas avaliações, com exceção de A12 e A14.

Esses dados replicam os achados de estudos que trabalharam com o ensino de leitura e escrita com diferentes populações, a partir de uma programação de ensino detaIhada em pequenos passos, com fornecimento de consequências imediatas para o acerto, ensino individualizado, respeitando o ritmo de aprendizagem individual de cada estudante (Benitez \& Domeniconi, 2016; Rose, Souza, Hanna, 1996; Melchiori, Souza, \& Rose, 2000; Sidman, 1971; Sidman, \& Cresson, 1973). 
Além de uma programação de ensino detalhada, outra discussão importante se refere ao local de aplicação (situação natural), assim como ao papel do aplicador em tal situação com os estudantes com DI e TEA. A aplicação no contexto da sala de aula ocorreu na presença do psicólogo-pesquisador, ao longo das atividades de língua portuguesa. Algumas ocorrências típicas do contexto de sala de aula podem ser listadas, como, interrupção da leitura pelo inspetor ou coordenador para emissão de recados, interrupção das atividades devido ao horário do recreio ou aula de educação física. No contexto das salas de educação especial e das residências os combinados foram realizados de acordo com a disponibilidade de cada agente educacional e contaram com entraves similares durante a aplicação, como, interrupção da atividade, devido à presença de outros profissionais (no contexto da sala de educação especial) ou colegas/parentes (na residência) e conversas paralelas sobre outros assuntos com o psicólogo-pesquisador.

Os aplicadores (professores e pais) foram expostos a uma capacitação sucinta que previa instrumentalizá-los para aplicar as tarefas de ensino, em seus ambientes naturais (sala de aula regular ou sala de educação especial ou residência), com acompanhamento do psicólogo-pesquisador. O arranjo das intervenções aplicadas por tais agentes, em conjunto com o psicólogo-pesquisador, favoreceu o processo de ensino das tarefas de leitura e escrita, o que gerou a aprendizagem de tais repertórios (com destaque aos dados do follow-up com o Grupo Experimental - Figura 2), devido ao fornecimento de serviços estruturados para cada estudante. Isso significa que o fornecimento de tais serviços, por um lado, gerou ocasião para aprendizagem ainda que introdutória, de repertórios complexos, com estudantes com DI e TEA e, por outro lado, contribuiu com a discussão sobre a inclusão escolar, a partir da disponibilização dos serviços para cada estudante, de acordo com o seu respectivo funcionamento e independência, na sala de aula comum com todos os estudantes (Mendes, 2010; Unesco, 1994), como uma forma de operacionalizar os achados descritos na Política de Educação Especial na perspectiva da Educação Inclusiva (Brasil, 2008).

Ao comparar a oferta de serviços (como, por exemplo, as intervenções propostas ao longo do estudo) disponibilizada para os estudantes do Grupo Experimental, com a ausência de tais serviços, referente aos estudantes do Grupo Controle, pode-se entender que o processo de inclusão escolar se torna insuficiente para todos os estudantes, se dependesse unicamente dos serviços (suporte/apoio) disponíveis para os alunos sem DI e sem TEA, como a participação na sala de aula comum, com um único professor. Isso denota a importância dos serviços dos diferentes agentes educacionais como uma proposta de efetivação do processo de inclusão escolar, a partir do sistema de suporte/apoio, em prol da aprendizagem dos conteúdos acadêmicos para todos os estudantes, ao invés de disseminar práticas que fomentam o assistencialismo e práticas integracionistas no âmbito educacional (Almeida, 2012; Benitez \& Domeniconi, 2014; Mendes, 2010; Vilaronga \& Mendes, 2014).

Nesse escopo de programação das intervenções e disponibilização de serviços, o papel do psicólogo-pesquisador foi fundamental para contribuir com o processo inclusivo dos estudantes com DI e TEA, a despeito das práticas segregadoras e integracionistas traçadas nas décadas anteriores, com esse público-alvo (Antunes, 2008; Guzzo, Mezzalira, Moreira, Tizzei, \& Silva Neto, 2010; Patto, 1993). Ao criar condições de monitoramento para as aplicações das intervenções conduzidas pelos agentes educacionais, com um mesmo objetivo, visando o ensino de leitura e escrita foi possível identificar uma forma de atuação inclusiva nesse cenário, ao invés de propagar práticas que visassem um modelo clínico, com enfoque psicométrico, centrado no sujeito, no lugar de considerar os fatores sociais, culturais, econômicos e, sobretudo, pedagógicos envolvidos com o processo de aprendizagem.

Assim sendo, os dados mostram uma forma de atuação do psicólogo no âmbito educacional que favorece a aprendizagem dos estudantes com TEA e DI, a partir do envolvimento de diferentes agentes educacionais, em uma perspectiva que envolve o processo pedagógico, os fatores sociais e simultaneamente, contempla o ritmo individual de aprendizagem de cada um deles, a partir de uma programação detalhada de ensino.

\section{Referências}

Almeida, M.A. (2012). O Caminhar da deficiência intelectual e classificação pelo sistema de suporte/apoio. In: Almeida, M. A. (Org.), Deficiência intelectual: realidade e ação (pp. 51-65). São Paulo: FDE.

Associação Americana de Psiquiatria [APA] (2013). Manual diagnóstico e estatístico de transtornos mentais - texto revisado (5a. ed.). Porto Alegre, RS: Artes Médicas.

Antunes, M.A.M. (2008). Psicologia escolar e educacional: história, compromissos e perspectivas. Psicologia Escolar e Educacional (ABRAPEE), 12(2), 469-475.

Baldaçara, L.; Nóbrega, L.P.C.; Tengan, S.K.; Maia, A.K. (2006). Hiperlexia em um caso de autismo e suas hipóteses. Revista de Psiquiatria Clínica, 33(5), 268-271.

Benitez, P.; Domeniconi, C. (2012). Verbalizações de familiares durante aprendizagem de leitura e escrita por deficientes intelectuais. Estudos de Psicologia, 29(4), 553-562.

Benitez, P.; Domeniconi, C. (2014). Capacitação de agentes educacionais: proposta de desenvolvimento de estratégias inclusivas. Revista Brasileira de Educação Especial, 30(3), 371-386.

Benitez, P.; Domeniconi, C. (2015). Inclusão escolar: o papel dos agentes educacionais brasileiros. Psicologia: Ciência e Profissão, 35, 1007-1023.

Benitez, P.; Domeniconi, C. (2016). Use of a computerized reading and writing teaching program for families of students with intellectual disabilities. The Psychological Record, 66, 127-138. 
Brasil. Ministério da Educação. Secretaria de Educação Especial. (2008). Política nacional de educação especial na perspectiva da educação inclusiva. Recuperado: 10 mai. 2012. Disponível: http:// portal.mec.gov.br/seesp/arquivos/pdf/politica.pdf.

Decreto $n^{\circ}$ 7.611, de 17 de novembro de 2011. (2011, 17 de novembro). Dispõe sobre a educação especial, o atendimento educacional especializado e dá outras providências. Brasília: Presidência da República. Recuperado: 26 dez. 2016. Disponível: http://www. planalto.gov.br/ccivil_03/_ato2011-2014/2011/decreto/d7611.htm>

Del Prette, Z.A.; Del Prette, A. (2008). Um sistema de categorias de habilidades sociais educativas. Paideia, 18(41), 517-530.

Guzzo, R.S.L.; Mezzalira, A.S.C.; Moreira, A.P.G.; Tizzei, R.P.; Silva Neto, W.M.F. (2010). Psicologia e educação no Brasil: uma visão da história e possibilidades nessa relação. Psicologia: Teoria e Pesquisa, 26(n. especial), 131-141.

Lovaas, O.I. (1987). Tratamiento conductual y funcionamiento educativo e intelectual normal en niños autistas. Journal of Consulting and Clinical Psychology, 1(55).

Melchiori, L.E.; Souza, D.G.; Rose, J.C. (2000). Reading, equivalence and recombination of units: a replication with students with different learning histories. Journal of Applied Behavior Analysis, 33, 97-100.

Mendes, E.G. (2010). Breve historia de la educación especial en Brasil. Educación y Pedagogía, 22(57), 93-109.

Miguel C.F.; Yang H.G.; Finn H.E.; Ahearn W.H. (2009). Establishing derived textual control in activity schedules with children with autism. Journal of Applied Behavior Analysis, 42, 703-709.

Patto, M.H.S. (1993). A produção do fracasso escolar. histórias de submissão e rebeldia. S. Paulo: TA Queiroz.

Reis, T.; Souza, D.G.; Rose, J.C. (2009). Avaliação de um programa para o ensino de leitura e escrita. Estudos Avaliação Educacional, 20, 425-449.
Rose, J.C.; Souza, D.G.; Hanna, E.S. (1996). Teaching reading and spelling: exclusion and stimulus equivalence. Journal of Applied Behavior Analysis, 29(4), 451-469.

Sidman, M. (1971). Reading and auditory-visual equivalence. Journal of Speech and Hearing Research, 14, 5-13.

Sidman, M.; Cresson, O. (1973) Reading and crosmodal transfer or stimulus equivalence in severe mental retardation. American Journal of Mental Deficiency, 77(1), 515-523.

Souza, D.G.; Rose, J.C.; Domeniconi, C. (2009). Applying relational operant's to reading and spelling. In: Rehfeldt, R.A.; BarnesHolmes, Y. (Orgs.). Derived relational responding: Applications for learners with autism and other developmental disabilities (pp.173207). Oakland, CA: New Harbinger Publications.

UNESCO (1994). Declaração de Salamanca. Recuperado: 10 mai. 2012. Disponível: http://portal.mec.gov.br/seesp/arquivos/pdf/salamanca.pdf

UNICEF (1990). Declaração mundial de educação para todos. Recuperado: 9 mai. 2014. Disponível: http://www.unicef.org/brazil/ pt/resources_10230.htm.

Vilaronga, C.A.R.; Mendes, E.G. (2014). Ensino colaborativo para o apoio à inclusão escolar: práticas colaborativas entre os professores. Revista Brasileira de Estudos Pedagógicos, 95(239), 139-151.

Wechsler, D.; Figueiredo, V.L.M. (2002). WISC-III: Escala de Inteligência Wechsler para crianças (Adaptação brasileira da $3^{\mathrm{a}}$ edição). São Paulo: Casa do Psicólogo.

Wehmeyer, M.L. (2003). Defining mental retardation and ensuring Access to the general curriculum. Education and Training in Developmental Disabilities, 38(3), 271-282.

Apoio financeiro: O presente trabalho recebeu financiamento da bolsa de estudos de Doutorado, referente à primeira autora, concedido pela FAPESP (Processo n²010/16701-0), com orientação da segunda autora, tal como financiamento do Benefício Complementar, referente à segunda autora, concedido pela FAPESP (Processo n 477068/2012-0).

\section{Sobre as autoras}

Priscila Benitez (priscila.benitez@ufabc.edu.br)

Doutora em Psicologia pela UFSCar, Professora adjunta no CMCC/UFABC. Universidade Federal do ABC. Santo André- SP. htttp://orcid.org/0000-0003-3501-7606

Camila Domeniconi (domeniconicamila@gmail.com)

Doutora em Educação Especial pela UFSCar, Professora adjunta no DEPsi/UFSCar. Universidade Federal de São Carlos. São Carlos- SP. htttp://orcid.org/0000-0003-0486-3543

(cc) EY License information: This is an open-access article distributed under the terms of the License (type CC-BY), which permits unrestricted use, distribution and reproduction in any medium, provided the original article is properly cited. 